\title{
Factorisation properties of the strong product
}

\author{
Gert de Cooman $^{1}$, Enrique Miranda ${ }^{2}$, and Marco Zaffalon ${ }^{3}$
}

\begin{abstract}
We investigate a number of factorisation conditions in the framework of sets of probability measures, or coherent lower previsions, with finite referential spaces. We show that the so-called strong product constitutes one way to combine a number of marginal coherent lower previsions into an independent joint lower prevision, and we prove that under some conditions it is the only independent product that satisfies the factorisation conditions. Keywords: coherent lower previsions, epistemic independence, strong independence, factorisation.
\end{abstract}

\section{Introduction}

In this paper, we investigate some relationships between the formalist and epistemic approaches to independence in a generalised setting that allows probabilities to be imprecisely specified. By formalist approach, we mean a way to construct an independent joint from given marginals that is based on requiring the joint to satisfy a number of mathematical properties, such as factorisation. An epistemic approach, on the other hand, uses judgements of equality between the marginal and conditional probability models to construct a joint from the marginals.

We will consider a finite number of logically independent variables $X_{n}$ assuming values in respective finite sets $\mathcal{X}_{n}, n \in N$, where $N$ denotes a finite index set. In an epistemic approach, we want to express that these variables are independent, in the sense that learning the values of some of them will not affect beliefs about the remaining ones. We base our analysis on the theory of coherent lower previsions, which are lower expectation functionals

Ghent University gert. decooman@ugent. be - Corresponding author. University of Oviedo, Dep. of Statistics and O.R. C-Calvo Sotelo, s/n 33007 Oviedo, Spain. Tel: (+34) 985102955, Fax: (+34) 985103354. mirandaenrique@uniovi.es · IDSIA zaffalon@idsia.ch 
equivalent to closed convex sets of probability mass functions. In the case of precise probability, we refer to an expectation functional as a linear prevision. We give a succinct introduction to the theory of coherent lower previsions in Section 2.

The real work begins in Section 3, where, in the formalist spirit, we introduce a number of factorisation conditions for coherent lower previsions, and establish relationships between them. In Section 4, we investigate a specific and fairly popular method for combining marginal coherent lower previsions into a joint lower prevision: the strong product. We show that this method satisfies all the formalist factorisation properties from Section 3, as well as two independence notions of an epistemic bent, called epistemic many-to-one and epistemic many-to-many independence. Moreover, we show that in certain cases the strong product is the only functional with these properties. Due to limitations of space, we have omitted the proofs of the main results.

\section{Coherent lower previsions}

We start with a brief introduction to the notions of the theory of coherent lower previsions; we refer to [8] for an in-depth study, and to [6] for a survey.

Consider a finite space $\mathcal{X}$. A gamble on $\mathcal{X}$ is a real-valued map $f: \mathcal{X} \rightarrow \mathbb{R}$. The set of all gambles on $\mathcal{X}$ is denoted by $\mathcal{L}(\mathcal{X})$. A linear prevision on $\mathcal{L}(\mathcal{X})$ is the expectation operator with respect to a probability on $\mathcal{X}$. A coherent lower prevision $\underline{P}$ on $\mathcal{L}(\mathcal{X})$ is the lower envelope of a closed and convex set of linear previsions, which we denote by $\mathcal{M}(\underline{P})$. One particular instance is the vacuous lower prevision with respect to a subset $A$ of $\mathcal{X}$, given by $\underline{P}^{A}(f)=\min _{\omega \in A} f(\omega)$ for all gambles $f$ on $\mathcal{X}$.

Next, consider a number of random variables $X_{n}, n \in N$, taking values in the respective finite sets $\mathcal{X}_{n}$. For every subset $J$ of $N$, we denote by $X_{J}$ the tuple of random variables (with one component for each $j \in J$ ) that takes values in the Cartesian product $\mathcal{X}_{J}=\times_{j \in J} \mathcal{X}_{j}$. We denote by $\mathcal{L}\left(\mathcal{X}_{J}\right)$ the set of all gambles on $\mathcal{X}_{J}$. We will frequently use the simplifying device of identifying a gamble $f_{J}$ on $\mathcal{X}_{J}$ with its cylindrical extension to $\mathcal{X}_{N}$, which is the gamble $f_{N}$ defined by $f_{N}\left(x_{N}\right)=f_{J}\left(x_{J}\right)$ for all $x_{N} \in \mathcal{X}_{N}$, where $x_{J}$ is the element of $\mathcal{X}_{J}$ consistent with $x_{N}$ (consistency means here that the components $x_{j}$ of $x_{J}$ and $x_{N}$ coincide for all $\left.j \in J\right)$.

Given two disjoint subsets $O$ and $I$ of $N$, we define a conditional lower prevision $\underline{P}_{O \cup I}\left(\cdot \mid X_{I}\right)$ as a special two-place function. For any $x_{I} \in \mathcal{X}_{I}$, $\underline{P}_{O \cup I}\left(\cdot \mid x_{I}\right)$ is a real-valued functional on the set $\mathcal{L}\left(\mathcal{X}_{O \cup I}\right)$ of all gambles on $\mathcal{X}_{O \cup I}$. For any gamble $f$ on $\mathcal{X}_{O \cup I}, \underline{P}_{O \cup I}\left(f \mid x_{I}\right)$ is the lower prevision of $f$, conditional on $X_{I}=x_{I}$. Moreover, the object $\underline{P}_{O \cup I}\left(f \mid X_{I}\right)$ is considered as a gamble on $\mathcal{X}_{I}$ that assumes the value $\underline{P}_{O \cup I}\left(f \mid x_{I}\right)$ in $x_{I}$.

We define, for any gamble $f$ on $\mathcal{X}_{O \cup I}$, the $\mathcal{X}_{I}$-support $S_{I}(f)$ of $f$ as $S_{I}(f)=\left\{x_{I} \in \mathcal{X}_{I}: \mathbb{I}_{\left\{x_{I}\right\}} f \neq 0\right\}$. Then a number of conditional linear pre- 
visions $P_{O_{j} \cup I_{j}}\left(\cdot \mid X_{I_{j}}\right)$ defined on the sets of gambles $\mathcal{L}\left(\mathcal{X}_{O_{j} \cup I_{j}}\right), j=1, \ldots, m$ are called coherent if for all $f_{j} \in \mathcal{L}\left(\mathcal{X}_{O_{j} \cup I_{j}}\right), j=1, \ldots, m$, there is some $j^{*} \in\{1, \ldots, m\}, x \in S_{I_{j^{*}}}\left(f_{j^{*}}\right)$ such that:

$$
\left[\sum_{j=1}^{m}\left(f_{j}-P_{O_{j} \cup I_{j}}\left(f_{j} \mid X_{I_{j}}\right)\right)\right]\left(x_{N}\right) \geq 0
$$

for some $x_{N} \in \mathcal{X}_{N}$ consistent with $x$. A number of conditional lower previsions $\underline{P}_{O_{j} \cup I_{j}}\left(\cdot \mid X_{I_{j}}\right)$ on $\mathcal{L}\left(\mathcal{X}_{O_{j} \cup I_{j}}\right), j=1, \ldots, m$ are called coherent if and only if they are the lower envelopes of some collection $\left\{P_{O_{j} \cup I_{j}}^{\lambda}\left(\cdot \mid X_{I_{j}}\right): \lambda \in \Lambda\right\}$ of coherent conditional linear previsions. In that case, they also satisfy in particular the property of weak coherence, which in this context holds if and only if there is some coherent lower prevision $\underline{P}_{N}$ on $\mathcal{L}\left(\mathcal{X}_{N}\right)$ such that $\underline{P}_{N}\left(\mathbb{I}_{\left\{x_{I_{j}}\right\}}\left[f-\underline{P}_{O_{j} \cup I_{j}}\left(f \mid x_{I_{j}}\right)\right]\right)=0$ for all $x_{I_{j}} \in \overline{\mathcal{X}}_{I_{j}}$ and all gambles $f$ on $\mathcal{X}_{O_{j} \cup I_{j}}, j \in\{1, \ldots, m\}$.

Finally, for any non-empty $R \subseteq N$, we denote by $\underline{P}_{R}$ (and by $\underline{P}_{r}$ if $R=$ $\{r\})$ the $\mathcal{X}_{R}$-marginal of a coherent lower prevision $\underline{P}_{N}$ on $\mathcal{L}\left(\mathcal{X}_{N}\right)$, given by $\underline{P}_{R}(f)=\underline{P}_{N}(f)$ for all gambles $f \in \mathcal{L}\left(\mathcal{X}_{R}\right)$.

\section{Factorisation conditions}

We begin our discussion by introducing a number of generalisations of the notion of an independent product of linear previsions. We have used the first of them in the context of our research on credal networks [2].

Definition 1. Consider a coherent lower prevision $\underline{P}_{N}$ on $\mathcal{L}\left(\mathcal{X}_{N}\right)$. We call this lower prevision

1. factorising if for all $o \in N$ and all non-empty $I \subseteq N \backslash\{o\}$, all $g \in \mathcal{L}\left(\mathcal{X}_{o}\right)$ and all non-negative $f_{i} \in \mathcal{L}\left(\mathcal{X}_{i}\right), i \in I, \underline{P}_{N}\left(f_{I} g\right)=\underline{P}_{N}\left(f_{I} \underline{P}_{N}(g)\right)$, where $f_{I}=\prod_{i \in I} f_{i}$.

2. strongly factorising if $\underline{P}_{N}(f g)=\underline{P}_{N}\left(f \underline{P}_{N}(g)\right)$ for all $g \in \mathcal{L}\left(\mathcal{X}_{O}\right)$ and $f \in \mathcal{L}\left(\mathcal{X}_{I}\right), f \geq 0$, where $I$ and $O$ are any disjoint proper subsets of $N$.

Our notion of factorisation when restricted to lower probabilities and events, is called strict factorisation in [7].

Next, we come to a property that V. Kuznetsov [5] first drew attention to:

Definition 2. Consider a coherent lower prevision $\underline{P}_{N}$ on $\mathcal{L}\left(\mathcal{X}_{N}\right)$. We call this lower prevision

1. Kuznetsov if $\underline{\bar{P}}_{N}\left(\prod_{n \in N} f_{n}\right)=\bigotimes_{n \in N} \underline{\bar{P}}_{n}\left(f_{n}\right)$ for all $f_{n} \in \mathcal{L}\left(\mathcal{X}_{n}\right), n \in N$.

2. strongly Kuznetsov if $\underline{\underline{P}}_{N}(f g)=\underline{\bar{P}}_{I}(f) \otimes \underline{\underline{P}}_{O}(g)$ for all $g \in \mathcal{L}\left(\mathcal{X}_{O}\right)$ and all $f \in \mathcal{L}\left(\mathcal{X}_{I}\right)$, where $I$ and $O$ are any disjoint proper subsets of $N$. 
Here $\boldsymbol{\nabla}$ is the (commutative and associative) interval product defined by:

$$
\begin{aligned}
{[a, b] \otimes[c, d] } & =\{x y: x \in[a, b] \text { and } y \in[c, d]\} \\
& =[\min \{a c, a d, b c, b d\}, \max \{a c, a d, b c, b d\}]
\end{aligned}
$$

for all $a \leq b$ and $c \leq d$ in $\mathbb{R}$, and $\underline{\bar{P}}(f)$ is the interval $[\underline{P}(f), \bar{P}(f)]$.

There are the following general relationships between these properties:

Proposition 1. Consider a coherent lower prevision $\underline{P}_{N}$ on $\mathcal{L}\left(\mathcal{X}_{N}\right)$. Then

$$
\begin{array}{ccc}
\underline{P}_{N} \text { is strongly Kuznetsov } & \Rightarrow \underline{P}_{N} \text { is strongly factorising } \\
\Downarrow & \Downarrow \\
\underline{P}_{N} \text { is Kuznetsov } & \Rightarrow & \underline{P}_{N} \text { is factorising. }
\end{array}
$$

What about the converse implications? We show in Example 2 that factorisation is not equivalent to being Kuznetsov, and that strong factorisation is not equivalent to being strongly Kuznetsov. In Example 3, we give an instance of a lower prevision that is factorising but not strongly factorising.

\section{The strong product}

The remainder of this paper is devoted to the study of a particular product of coherent lower previsions, called the strong product, which also appears under the name type-1 product [8, Section 9.3.5]. Our name for it seems to go back to Cozman [1]. If we have coherent lower previsions $\underline{P}_{n}$ on $\mathcal{L}\left(\mathcal{X}_{n}\right)$, then $\left[8\right.$, Section 9.3.5] their strong product $\underline{S}_{N}=\times_{n \in N} \underline{P}_{n}$ is defined by

$$
\begin{aligned}
\underline{S}_{N}(f) & =\inf \left\{\times_{n \in N} P_{n}(f):(\forall n \in N) P_{n} \in \mathcal{M}\left(\underline{P}_{n}\right)\right\} \\
& =\inf \left\{\times_{n \in N} P_{n}(f):(\forall n \in N) P_{n} \in \operatorname{ext}\left(\mathcal{M}\left(\underline{P}_{n}\right)\right)\right\}
\end{aligned}
$$

for every $f \in \mathcal{L}\left(\mathcal{X}_{N}\right)$, where $\times_{n \in N} P_{n}$ is the usual independent product of the considered linear previsions. The strong product of lower previsions satisfies the following marginalisation and associativity properties.

Proposition 2. Consider coherent lower previsions $\underline{P}_{n}$ on $\mathcal{L}\left(\mathcal{X}_{n}\right), n \in N$.

1. For any non-empty subset $R$ of $N, \underline{S}_{R}$ is the $\mathcal{X}_{R}$-marginal of $\underline{S}_{N}$;

2. $\operatorname{ext}\left(\mathcal{M}\left(\underline{S}_{N}\right)\right)=\left\{\times_{n \in N} P_{n}:(\forall n \in N) P_{n} \in \operatorname{ext}\left(\mathcal{M}\left(\underline{P}_{n}\right)\right)\right\}$;

3. For any partition $N_{1}$ and $N_{2}$ of $N, \underline{S}_{N}=\underline{S}_{N_{1}} \times \underline{S}_{N_{2}}$.

We can deduce from the second statement that the infima in Eqs. (1) and (2) are actually minima. This allows us to deduce that the strong product of lower previsions satisfies all the conditions introduced in Section 3. 
Proposition 3. The strong product $\underline{S}_{N}$ is strongly Kuznetsov, and therefore also Kuznetsov, strongly factorising and factorising.

This generalises a result established for the case of two variables by Cozman [1]. It also guarantees that the strong product satisfies the weak law of large numbers established in [3].

As a next step, we establish a tighter relationship between the strong product and the epistemic approach to independence. Consider two disjoint proper subsets $I$ and $O$ of $N$. We say that a subject judges that $X_{I}$ is epistemically irrelevant to $X_{O}$ when he assumes that learning which value $X_{I}$ assumes in $\mathcal{X}_{I}$ will not affect his beliefs about $X_{O}$. We say that a subject judges the variables $X_{n}, n \in N$ to be epistemically many-to-many independent when he judges for any disjoint proper subsets $I$ and $O$ of $N$ that $X_{I}$ is epistemically irrelevant to $X_{O}$. If our subject has a coherent lower prevision $\underline{P}_{N}$ on $\mathcal{L}\left(\mathcal{X}_{N}\right)$, and he makes such an assessment, then he can infer from his joint model $\underline{P}_{N}$ a family of conditional models

$$
\mathcal{I}\left(\underline{P}_{N}\right)=\left\{\underline{P}_{O \cup I}\left(\cdot \mid X_{I}\right): I \text { and } O \text { disjoint proper subsets of } N\right\},
$$

where $\underline{P}_{O \cup I}\left(\cdot \mid X_{I}\right)$ is a coherent lower prevision on $\mathcal{L}\left(\mathcal{X}_{O \cup I}\right)$ that is given by:

$$
\underline{P}_{O \cup I}\left(h \mid x_{I}\right)=\underline{P}_{N}\left(h\left(\cdot, x_{I}\right)\right) \text { for all } h \in \mathcal{L}\left(\mathcal{X}_{O \cup I}\right) \text { and all } x_{I} \in \mathcal{X}_{I} .
$$

Definition 3. A coherent lower prevision $\underline{P}_{N}$ on $\mathcal{L}\left(\mathcal{X}_{N}\right)$ is called many-tomany independent if it is coherent with the family of conditional lower previsions $\mathcal{I}\left(\underline{P}_{N}\right)$, and in that case it is also called a many-to-many independent product of its marginal coherent lower previsions $\underline{P}_{n}$ on $\mathcal{L}\left(\mathcal{X}_{n}\right), n \in N$.

In a similar way, we say that a subject judges the variables $X_{n}, n \in N$ to be epistemically many-to-one independent when he assumes that learning the value of any number of these variables will not affect his beliefs about any single other. If our subject has a coherent lower prevision $\underline{P}_{N}$ on $\mathcal{L}\left(\mathcal{X}_{N}\right)$, and he makes such an assessment, then he can infer from his joint model $\underline{P}_{N}$ a family of conditional models

$$
\mathcal{N}\left(\underline{P}_{n}, n \in N\right)=\left\{\underline{P}_{\{o\} \cup I}\left(\cdot \mid X_{I}\right): o \in N \text { and } I \subseteq N \backslash\{o\}\right\},
$$

where $\underline{P}_{\{o\} \cup I}\left(\cdot \mid X_{I}\right)$ is a coherent lower prevision on $\mathcal{L}\left(\mathcal{X}_{\{o\} \cup I}\right)$ given by:

$$
\underline{P}_{\{\circ\} \cup I}\left(h \mid x_{I}\right)=\underline{P}_{N}\left(h\left(\cdot, x_{I}\right)\right)=\underline{P}_{o}\left(h\left(\cdot, x_{I}\right)\right)
$$

for all gambles $h$ on $\mathcal{X}_{\{o\} \cup I}$ and all $x_{I} \in \mathcal{X}_{I}$.

Definition 4. A coherent lower prevision $\underline{P}_{N}$ on $\mathcal{L}\left(\mathcal{X}_{N}\right)$ is called many-toone independent if it is coherent ${ }^{1}$ with the family $\mathcal{N}\left(\underline{P}_{n}, n \in N\right)$, and in

\footnotetext{
${ }^{1}$ Actually, thanks to [4, Proposition 10], weak coherence suffices here.
} 
that case it is also called a many-to-one independent product of its marginal coherent lower previsions $\underline{P}_{n}$ on $\mathcal{L}\left(\mathcal{X}_{n}\right), n \in N$.

A basic coherence result [8, Theorem 7.1.6] states that taking lower envelopes of a family of coherent conditional lower previsions again produces coherent conditional lower previsions. Using this, we can deduce that there always is at least one many-to-many (and therefore also many-to-one) independent product: the strong product.

Proposition 4. Consider arbitrary coherent lower previsions $\underline{P}_{n}$ on $\mathcal{L}\left(\mathcal{X}_{n}\right)$, $n \in N$. Then their strong product $\times_{n \in N} \underline{P}_{n}$ is a many-to-many and many-toone independent product of the marginal lower previsions $\underline{P}_{n}$.

The strong product is not in general the only many-to-one (or many-to-many) independent product of given marginals: there usually are an infinity of them. The smallest is called the independent natural extension. We have studied it in detail in another paper [4]. It does not coincide in general with the strong product [8, Section 9.3.4]. Neither is the strong product generally the greatest many-to-one independent product of its marginals:

Example 1. Consider $\mathcal{X}_{1}=\mathcal{X}_{2}=\{0,1\}$, and let $\underline{P}_{1}$ and $\underline{P}_{2}$ be the vacuous lower previsions on $\mathcal{L}\left(\mathcal{X}_{1}\right)$ and $\mathcal{L}\left(\mathcal{X}_{2}\right)$, respectively. Then the strong product $\underline{S}_{\{1,2\}}=\underline{P}_{1} \times \underline{P}_{2}$ is the vacuous lower prevision on $\mathcal{L}\left(\mathcal{X}_{\{1,2\}}\right)$.

Let $\underline{Q}_{\{1,2\}}$ be the vacuous lower prevision relative to $\{(0,0),(1,1)\}$, which clearly strictly dominates the strong product $\underline{S}_{\{1,2\}}$. To see that it is also a many-to-one independent product of the marginals $\underline{P}_{1}$ and $\underline{P}_{2}$, it suffices [cf. footnote 1] to show that $\underline{Q}_{\{1,2\}}\left(\mathbb{I}_{\left\{x_{1}\right\}}\left[g_{2}-\underline{P}_{2}\left(g_{2}\right)\right]\right)=0$ for all $x_{1} \in \mathcal{X}_{1}$ and all $g_{2} \in \mathcal{L}\left(\mathcal{X}_{2}\right)$ [the case $x_{2} \in \mathcal{X}_{2}$ and all $g_{1} \in \mathcal{L}\left(\mathcal{X}_{1}\right)$ is symmetric]. But $\underline{Q}_{\{1,2\}}\left(\mathbb{I}_{\left\{x_{1}\right\}}\left[g_{2}-\underline{P}_{2}\left(g_{2}\right)\right]\right)$ is equal to

$$
\min \left\{\mathbb{I}_{\left\{x_{1}\right\}}(0)\left[g_{2}(0)-\underline{P}_{2}\left(g_{2}\right)\right], \mathbb{I}_{\left\{x_{1}\right\}}(1)\left[g_{2}(1)-\underline{P}_{2}\left(g_{2}\right)\right]\right\}=0,
$$

since both $\mathbb{I}_{\left\{x_{1}\right\}}(0)\left[g_{2}(0)-\underline{P}_{2}\left(g_{2}\right)\right]$ and $\mathbb{I}_{\left\{x_{1}\right\}}(1)\left[g_{2}(1)-\underline{P}_{2}\left(g_{2}\right)\right]$ are nonnegative, and at least one of these numbers is zero.

We have shown in [4, Proposition 13] that when $N=\{1,2\}$ and one of the marginals is vacuous, the strong product coincides with the independent natural extension and is therefore the smallest independent product of the given marginals. Example 1 shows it is not the only many-to-one independent product if one of the marginals is vacuous. Yet, it is the only one factorising:

Proposition 5. Let $\underline{P}_{1}^{A_{1}}$ be the vacuous lower prevision on $\mathcal{L}\left(\mathcal{X}_{1}\right)$ relative to the non-empty set $A_{1} \subseteq \mathcal{X}_{1}$, and let $\underline{P}_{2}$ be any coherent lower prevision on $\mathcal{L}\left(\mathcal{X}_{2}\right)$. Then any factorising product $\underline{P}$ of these marginals satisfies

$$
\underline{P}(f)=\left(\underline{P}_{1}^{A_{1}} \times \underline{P}_{2}\right)(f)=\min _{x_{1} \in A_{1}} \underline{P}_{2}\left(f\left(x_{1}, \cdot\right)\right) \text { for all gambles } f \in \mathcal{L}\left(\mathcal{X}_{\{1,2\}}\right) \text {. }
$$


We now come to the notion of external additivity:

Definition 5. Consider a coherent lower prevision $\underline{P}_{N}$ on $\mathcal{L}\left(\mathcal{X}_{N}\right)$. It is called externally additive if for all non-empty $R \subseteq N$ and all gambles $f_{r}$ on $\mathcal{X}_{r}$, $r \in R, \underline{P}_{N}\left(\sum_{r \in R} f_{r}\right)=\sum_{r \in R} \underline{P}_{N}\left(f_{r}\right)$, and strongly externally additive if $\underline{P}_{N}(f+g)=\underline{P}_{N}(f)+\underline{P}_{N}(g)$ for all $f \in \mathcal{L}\left(\mathcal{X}_{I}\right), g \in \mathcal{L}\left(\mathcal{X}_{O}\right)$, where $I$ and $O$ are any disjoint proper subsets of $N$.

Clearly, strong external additivity implies external additivity. Cozman calls the latter summation independence, and shows [1, Theorem 1] that the strong product is externally additive for the case of two variables. We generalise this by proving that the strong product is strongly externally additive.

Proposition 6. Consider arbitrary coherent lower previsions $\underline{P}_{n}, n \in N$. Then their strong product $\underline{S}_{N}$ is strongly externally additive.

We have established in [4, Theorem 5] that the independent natural extension is strongly factorising. We now show that it is not Kuznetsov in general:

Example 2. Consider random variables $X_{1}, X_{2}$ assuming values in $\{0,1\}$, and let their marginal lower previsions be given by

$$
\underline{P}_{j}\left(f_{j}\right)=\frac{1}{2} f_{j}(0)+\frac{2}{5} f_{j}(1)+\frac{1}{10} \min \left\{f_{j}(0), f_{j}(1)\right\} \text { for all } f_{j} \in \mathcal{X}_{j}
$$

for $j=1,2$ (these are linear-vacuous mixtures, and hence coherent $[8$, Section 2.9.2]). Consider the gambles $f=\mathbb{I}_{\{0\}}-\mathbb{I}_{\{1\}}$ on $\mathcal{X}_{1}$ and $g=\mathbb{I}_{\{0\}}-\mathbb{I}_{\{1\}}$ on $\mathcal{X}_{2}$. Then $\underline{P}_{1}(f)=\underline{P}_{2}(g)=0$ and $\bar{P}_{1}(f)=\bar{P}_{2}(g)=1 / 5$. As a consequence, $\underline{\underline{P}}_{1}(f) \otimes \underline{\underline{P}}_{2}(g)=[0,1 / 25]$, whereas their independent natural extension assumes [8, Example 9.3.4] the value $\underline{E}_{\{1,2\}}(f g)=-1 / 11$. This shows that the independent natural extension $\underline{E}_{\{1,2\}}$, which is factorising, is not Kuznetsov. Moreover, in this example where $N=\{1,2\}$, factorisation is equivalent to strong factorisation, and being Kuznetsov to being strongly Kuznetsov.

A convex combination of many-to-one independent products of the same marginals is again a many-to-one independent product of these marginals [4, Proposition 8]. A similar result holds for factorising or Kuznetsov lower previsions. We use these ideas to construct the following counterexample, which shows that a many-to-one independent product is not necessarily many-tomany, and that a factorising lower prevision need not be strongly factorising.

Example 3. Let $N=\{1,2,3\}$. Consider random variables $X_{j}$ assuming values in $\mathcal{X}_{j}=\{0,1\}$ for $j=1,2,3$. Let the corresponding marginal lower previsions be given by

$$
\underline{P}_{j}\left(f_{j}\right)=\frac{1}{2} f_{j}(0)+\frac{2}{5} f_{j}(1)+\frac{1}{10} \min \left\{f_{j}(0), f_{j}(1)\right\} \text { for all } f_{j} \in \mathcal{L}\left(\mathcal{X}_{j}\right)
$$

for $j=1,2,3$. Let $\underline{E}_{N}$ be their independent natural extension and $\underline{S}_{N}$ their strong product, and define $\underline{Q}_{N}$ on $\mathcal{L}\left(\mathcal{X}_{N}\right)$ as $\underline{Q}_{N}=1 / 2\left(\underline{E}_{N}+\underline{S}_{N}\right)$. It follows 
from Propositions 3, 4 and [4, Proposition 8] that $\underline{Q}_{N}$ is factorising and a many-to-one independent product. We are going to prove that $\underline{Q}_{N}$ is neither a many-to-many independent product nor strongly factorising.

Consider the conditional lower prevision $\underline{Q}_{N}\left(\cdot \mid X_{3}\right)$ defined from the joint lower prevision $\underline{Q}_{N}$ using the epistemic irrelevance of $X_{3}$ to $X_{\{1,2\}}$. In order to show that $\underline{Q}_{N}$ is not a many-to-many independent product, it suffices to show that it is not weakly coherent with $\underline{Q}_{N}\left(\cdot \mid X_{3}\right)$. Consider the event $A$ that $X_{1}=$ $X_{2}$, and the corresponding indicator gamble $g=\mathbb{I}_{A}$ on $\mathcal{X}_{\{1,2\}}$. It follows from [8, Example 9.3.4] that $\underline{E}_{N}(A)=5 / 11$ and $\underline{S}_{N}(A)=1 / 2$, so $\underline{Q}_{N}(A)=21 / 44$. Let $x_{3}=0$. Since both $\underline{E}_{N}$ and $\underline{S}_{N}$ are strongly factorising (by Proposition 3 and [4, Theorem 5]), we see that $\underline{E}_{N}\left(\mathbb{I}_{\left\{x_{3}\right\}}\left[g-Q_{N}(g)\right]\right)=-3 / 220$ whereas $\underline{S}_{N}\left(\mathbb{I}_{\left\{x_{3}\right\}}\left[g-\underline{Q}_{N}(g)\right]\right)=1 / 88$, and then $\underline{Q}_{N}\left(\mathbb{I}_{\left\{x_{3}\right\}}\left[\bar{g}-\underline{Q}_{N}(g)\right]\right)=-1 / 440<0$. This shows that $\underline{Q}_{N}$ is not weakly coherent with $\underline{Q}_{N}\left(\cdot \mid X_{3}\right)$, and also that it is not strongly factorising.

\section{Conclusions}

The strong product satisfies all factorisation properties introduced in this paper, and it is a many-to-many independent product of the given marginals. In this sense, it satisfies more factorisation properties than the independent natural extension we studied in another paper [4], because the latter need not be Kuznetsov. Topics of future research could be the generalisation of these results towards infinite spaces as well as the study of the sets of all independent products in some interesting particular cases.

Acknowledgements Work supported by SBO project 060043 of the IWT-Vlaanderen, by projects TIN2008-06796-C04-01 and MTM2007-61193, and by Swiss NSF grants n. 200020$116674 / 1$ and 200020-121785/1.

\section{References}

1. Cozman F (2001) Constructing sets of probability measures through Kuznetsov's independence condition. In De Cooman, G, Fine, T. and Seidenfeld, T. (eds.) Proceedings of ISIPTA '01. Shaker Publishing, Maastricht.

2. De Cooman, G, Hermans, F, Antonucci, A and Zaffalon, M (2010). Epistemic irrelevance in credal nets: the case of imprecise Markov trees. Int. J. of App. Reasoning, accepted for publication. Extended version of a paper in the proceedings of ISIPTA'09.

3. De Cooman G and Miranda E (2008) J. of Stat. Plann. Inference, 138(8):2409-2432.

4. De Cooman G and Miranda E and Zaffalon M (2010) Independent natural extension. Proceedings of IPMU-2010.

5. Kuznetsov V (1991) Interval Statistical Methods. Radio i Svyaz Publ. In Russian.

6. Miranda E (2008) Int. J. of App. Reasoning 48(2):628-658.

7. Vicig P (2000), Int. J. of App. Reasoning 24(3):235-250.

8. Walley P (1991) Statistical Reasoning with Imprecise Probabilities. Chapman and Hall, London. 